\title{
Propriedade do divergente para campos vetoriais não diferenciáveis em duas zonas.
}

\author{
João Medrado, \\ Instituto de Matemática e Estatística, UFG \\ 74001-970, Goiânia, GO \\ E-mail: medrado@ufg.br, \\ Joan Torregrosa \\ UAB - Departament de Matemátiques \\ Barcelona, Espanha \\ E-mail: torre@mat.uab.cat.
}

Resumo: Neste trabalho estendemos a propriedade do divergente para os campos vetoriais não diferenciáveis definidos em duas zonas.

Palavras-chave: campos vetoriais não diferenciáveis, estabilidade, órbita periódica

Os modelos usados em muito problemas relacionados com engenharia, biologia, Teoria do Controle, design de circuitos elétricos, sistemas mecânicos, ciências econômicas e medicina, geralmente envolvem campos vetoriais não diferenciáveis. Uma ferramenta importante e simples para a descrição destes modelos é o estudo de campos vetoriais lineares definidos por partes. Em $[1,7]$ tem-se uma boa coleção de modelos e aplicações reais envolvendo esta classe de campos vetoriais. Tipicamente, esta classe de sistemas é obtida usando dois ou mais campos vetoriais lineares que são definidos em diferentes regiões separadas por conjuntos de descontinuidade. Em particular, um circuito tendo uma chave ideal pode ser modelado com um sistema linear planar por partes, veja seção 1.1.7 de [1].

Interessante observar que os sistemas diferenciais planares lineares são completamente estudados usando somente álgebra linear e eles não apresentam órbitas periódicas isoladas, chamados ciclos limite. Entretanto, ao estudarmos estes sistemas definidos em duas zonas, encontramos hoje na literatura exemplos com 4 ciclos limite, ou seja, os problemas envolvendo estes campos vetoriais ensejam um tratamento diferenciado e novo.

A classificação dos diferentes retratos de fase de campos vetoriais não diferenciáveis ou a determinação do número máximo de ciclos limite são problemas abertos, mesmo quando o número de regiões é pequeno, dois em nosso caso. A existência de pontos singulares reais e/ou virtuais, conexão de separatrizes, órbitas periódicas isoladas, são problemas novos neste contexto, e mais, têm-se uma maior riqueza de retratos de fase mesmo para a classe de campos vetoriais lineares por partes em relação ao campos vetoriais lineares definidos em uma região.

Uma importante ferramenta estudar a existência ou estabilidade de uma órbita periódica de campo vetorial diferenciável, é o cálculo da integral do divergente de um campo vetorial ao longo de uma órbita periódica, veja [8]. Para campos vetoriais não diferenciáveis em duas zonas, esta propriedade não pode ser usada quando a órbita periódica intersecta a linha de descontinuidade e desta forma para estudarmos a estabilidade destas órbitas para campos vetoriais não diferenciáveis, precisamos estender este resultado.

Definamos de forma mais precisa um campo vetorial não diferenciável em duas zonas. Para isto, considere $h: \mathbb{R}^{2} \rightarrow \mathbb{R}$ uma função e denotemos por $\Sigma=h^{-1}(0)$ e por $\Sigma^{ \pm}=\{ \pm h(x, y)>0\}$, as 
regiões distinguidas por esta curva no plano. Com esta notação, consideramos o campo vetorial não diferenciável em duas zonas $Z=(X, Y)$ definido por

$$
Z(q)= \begin{cases}X(q), & h(q)>0 \\ Y(q), & h(q)<0\end{cases}
$$

onde $X, Y$ são campos vetoriais diferenciáveis planares. O campo vetorial $Z$ está definido em $\Sigma$ seguindo a convenção de Filippov apresentada em [9]. Geometricamente, os pontos em $\Sigma$ onde os ambos campos vetoriais $X$ and $Y$ simultaneamente apontam para dentro ou para fora de $\Sigma$ definem a região de deslize ou escape, e o complemento em $\Sigma$ define a região de costura (veja Figura 1). De fato, a fronteira destas regiões é defina pelos pontos de tangência das órbitas de $X$ e $Y \operatorname{com} \Sigma$.

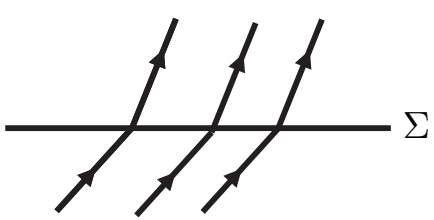

Pontos de costura

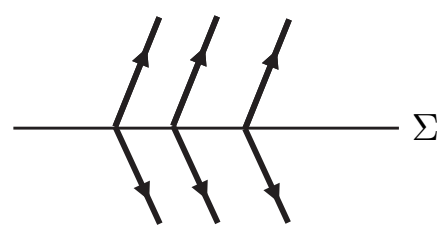

Pontos de escape

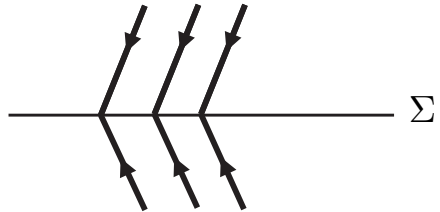

Pontos de deslize

Figura 1: Pontos de costura, escape e deslize.

Mais especificamente, os pontos de costura de $Z=(X, Y)$ em $\Sigma=h^{-1}(0)$ satisfazem $X h(p)$. $Y h(p)>0$, onde $X h$ denota a derivada da função $h$ na direção de $X$ i.e., $X h(p)=\langle\nabla h(p), X(p)\rangle$. O ponto $p$ em $\Sigma$ é um ponto de tangência de $X($ resp. $Y$ ) se $X h(p)=0($ resp. $Y h(p)=0)$ e dizemos que $p \in \Sigma$ é um $\Sigma$-ponto singular de $Z$ se $p \in \Sigma$ é um ponto de tangência ou ponto singular $(X(p)=0$ ou $Y(p)=0)$ de $X$ ou $Y$. Dizemos que o ponto $p \in \Sigma$ é uma dobra invisível (resp. visível) de $X$ se $p$ é um ponto de tangência de $X$ e $X^{2} h(p)<0\left(\right.$ resp. $\left.Y^{2} h(p)>0\right)$. A ordem do contato entre as órbitas de $X$ e a curva $\Sigma$ nos dá a característica da tangência.

\section{Existência, não existência e estabilidade de ciclos limite}

Nesta seção, obtemos resultados sobre sistemas diferenciais em duas zonas $\tilde{Z}=\left(X^{+}, X^{-}\right)$, onde $\phi$ é uma função do plano e 0 é um valor regular com $\tilde{\Sigma}=\phi^{-1}(0)$ e os campos vetoriais $X^{+}$e $X^{-}$estão definidos em $\tilde{\Sigma}^{+}=\phi^{-1}(0)>0$ e $\tilde{\Sigma}^{-}=\phi^{-1}(0)<0$, respectivamente.

Seja $\gamma(t, p)=(\varphi(t), \psi(t))$ uma solução do campo vetorial planar $\tilde{X}(x, y)=(f(x, y), g(x, y))$ tal que $\gamma(0, p)=p$ e $\tilde{\Sigma}=(\alpha(s), \beta(s)), s \in I \subset \mathbb{R}$ seja uma curva regular dada por $\phi^{-1}(0)$, onde $\phi$ é uma função do plano e 0 é um valor regular de $\tilde{\Sigma}$. Sejam ainda $\tilde{\Sigma}_{0}, \tilde{\Sigma}_{1} \subset \tilde{\Sigma}$ seções transversais a $\gamma$ em $p=\gamma(0) \in \tilde{\Sigma}_{0}$ e $q=\gamma(\tau) \in \tilde{\Sigma}_{1}$, respectivamente, onde $\tau$ é o menor valor tal que $\gamma(t, 0) \cap \tilde{\Sigma}_{1}, t>0$. (veja Figura 1 )

Em acordo com o estudo da estabilidade de uma órbita periódica descrito em [2], nós temos o seguinte resultado.

Proposition 1.1. Seja П a aplicação de Poincaré entre as seções $\tilde{\Sigma}_{0}$ e $\tilde{\Sigma}_{1}$ da órbita $\gamma(t, p)=$ $(\varphi(t), \psi(t)), t \in[0, \tau]$ de $\tilde{X}(x, y)=(f(x, y), g(x, y))$. Então a derivada de $\Pi$ em $p \in \tilde{\Sigma}_{0} \cap \gamma$ é dada por

$$
\Pi^{\prime}(p)=\frac{\nabla(0,0)}{\nabla(\tau, 0)} \exp \left(\int_{0}^{\tau} \operatorname{div} \tilde{X}(\gamma(s)) d s\right)
$$

onde $\nabla(t, 0)=\left|\begin{array}{cc}\varphi^{\prime}(t) & \psi^{\prime}(t) \\ \alpha^{\prime}(0) & \beta^{\prime}(0)\end{array}\right|$

A seguir, estendemos este resultado para campos vetoriais não diferenciáveis em duas zonas, $Z=\left(X^{+}, X^{-}\right)$. 


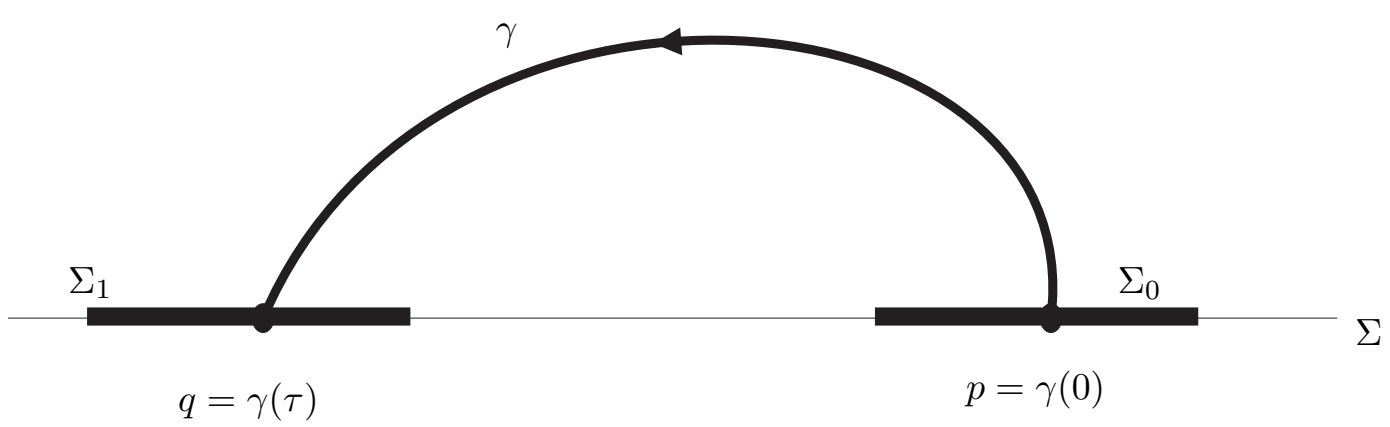

Figura 2: Aplicação de Poincaré.

Theorem 1.2. Considere $\tilde{Z}=\left(X^{+}, X^{-}\right)=\left(\left(f^{+}, g^{+}\right),\left(f^{-}, g^{-}\right)\right)$um $\tilde{\Sigma}$-campo vetorial em duas zonas em $\mathbb{R}^{2}$ e $\gamma=\gamma^{+} \cup \gamma^{-}$uma $\Sigma$-órbita periódica por $p^{ \pm} \in \tilde{\Sigma}$. Então a derivada da aplicação de Poincaré vinculada a $\gamma$ em $p \in \sigma$ é dada por

$$
\Pi^{\prime}(p)=\frac{X^{+} \phi(p)}{X^{-} \phi(p)} \frac{X^{-} \phi(q)}{X^{+} \phi(q)} \exp \int_{\gamma} \operatorname{div} \tilde{Z},
$$

onde $\operatorname{div} \tilde{Z}=\operatorname{div} X^{ \pm}$on $\Sigma^{ \pm}$.

Demonstração. Sejam $\Sigma_{0}, \Sigma_{1} \subset \Sigma$ seções tranversais a $\gamma$ em $p$ e $q$, respectivamente. Sejam ainda $\Pi^{ \pm}$as aplicações de Poincaré associadas a $\gamma^{ \pm}$, respectively. Definimos a aplicação de Poincaré associada a $\gamma$ pela composição $\Pi=\Pi^{-}\left(\Pi^{+}\right)$. Note que a derivada de $\Pi$ em $p$ é obtida multiplicando as derivadas de $\Pi^{+}$e $\Pi^{-}$em $p$.

Considere uma parametrização de $\tilde{\Sigma}$ dada por $\alpha(s), \beta(s), s \in I \subset \mathbb{R}$. Temos que $\phi(\alpha(s), \beta(s))=$ 0 e

$$
\left(\alpha^{\prime}(s), \beta^{\prime}(s)\right)=\lambda\left(-\phi_{y}, \phi_{x}\right)
$$

para uma constante dada $\lambda$ a qual depende da parametrização. Assim, da Proposição 1.1, sabemos que

$$
\left(\Pi^{ \pm}\right)^{\prime}\left(p^{ \pm}\right)=\frac{\left|\begin{array}{cc}
f^{ \pm}\left(p^{ \pm}\right) & g^{ \pm}\left(p^{ \pm}\right) \\
-\phi_{y}\left(p^{ \pm}\right) & \phi_{x}\left(p^{ \pm}\right)
\end{array}\right|}{\left|\begin{array}{cc}
f^{ \pm}\left(p^{\mp}\right) & g^{ \pm}\left(p^{\mp}\right) \\
-\phi_{y}\left(p^{\mp}\right) & \phi_{x}\left(p^{\mp}\right)
\end{array}\right|} \exp \int_{\gamma^{ \pm}} \operatorname{div}\left(X^{ \pm}\right) .
$$

Observamos que denotamos por $p^{+}$e $p^{-}$ao invés de $p$ and $q$ para que tenhamos uma expressão unificada das derivadas. Note ainda que a constante $\lambda$ desaparece porque ela é fator comum no numerador e também no denominador da fração acima.

Finalizamos a prova, mostrando que

$$
\left|\begin{array}{cc}
f^{+}(p) & g^{+}(p) \\
-\phi_{y}(p) & \phi_{x}(p)
\end{array}\right|=<X^{+}, \nabla \phi(p)>=X^{+} \phi(p) .
$$

Equivalentemente para $X^{-} \phi(p)$ e $X^{ \pm} \phi(q)$.

Por uma mudança de coordenadas, podemos considerar localmente que a linha de descontinuidade $\Sigma$ seja uma reta. Desta forma, geralmente considera-se então $\Sigma=\left\{(x, y) \in \mathbb{R}^{2} \mid y=0\right\}$. Assim, temos como consequência deste teorema, que podemos escrever a derivada da aplicação de Poincaré de uma órbita periódica em uma forma mais simples dada no seguinte Corolário. 
Corollary 1.3. Considere $\tilde{Z}=\left(X^{+}, X^{-}\right)$um $\tilde{\Sigma}$-campo vetorial não diferenciável em duas zonas como no Teorema 1.2

(i) $\operatorname{Se} \phi(x, y)=y$ então

$$
\Pi^{\prime}\left(p^{+}\right)=\frac{g^{+}(p)}{g^{-}(p)} \frac{g^{-}(q)}{g^{+}(q)} \exp \int_{\gamma} \operatorname{div} \tilde{Z}
$$

(ii) Se $\tilde{Z}$ é contínua na segunda coordenada então

$$
\Pi^{\prime}(p)=\exp \int_{\gamma} \operatorname{div} \tilde{Z}
$$

Considerando o campo vetorial $Z=(X, Y)$ definido em duas zonas, onde $X$ and $Y$ são campos vetoriais lineares, então como não temos ciclos limite em $\Sigma^{+}$ou $\Sigma^{-}$, temos que os ciclos limite de $Z$ devem intersectar $\Sigma$, ou seja, os ciclos limite devem envolver o $\Sigma$-ponto singular. Temos o seguinte resultado.

Proposition 1.4. Todo ciclo limite de um campo vetorial linear por partes em duas zonas tem um $\Sigma$-ponto singular em seu interior.

O Corolário 1.3.(ii) provê as condições para determinar a estabilidade de um ciclo limite. Em particular, a segunda parte estabelece que o Critério de Dulac (veja em [8]), para a não existência de soluções periódicas é também válida.

\section{Referências}

[1] V. Acary, O. Bonnefon, and B. Brogliato. Nonsmooth modeling and simulation for switched circuits, volume 69 of Lecture Notes in Electrical Engineering. Springer, Dordrecht, 2011.

[2] A. A. Andronov, E. A. Leontovich, I. I. Gordon, and A. G. Maĭer. Theory of bifurcations of dynamic systems on a plane. Halsted Press [A division of John Wiley \& Sons], New York-Toronto, Ont., 1973.

[3] J. C. Artés, J. Llibre, J. C. Medrado, and M. A. Teixeira. Piecewise linear differential systems with two real saddles. Math. Comput. Simulation, 95:13-22, 2013.

[4] C. A. Buzzi, J. C. Medrado, and M. A. Teixeira. Generic bifurcation of refracted systems. Adv. Math., 234:653-666, 2013.

[5] C. A. Buzzi, C. Pessoa, and J. Torregrosa. Piecewise linear perturbations of a linear center. Discrete Contin. Dyn. Syst., 33(9):3915-3936, 2013.

[6] B. Coll, A. Gasull, and R. Prohens. Degenerate Hopf bifurcations in discontinuous planar systems. J. Math. Anal. Appl., 253(2):671-690, 2001.

[7] M. di Bernardo, C. J. Budd, A. R. Champneys, and P. Kowalczyk. Piecewise-smooth dynamical systems. Theory and applications, volume 163 of Applied Mathematical Sciences. Springer-Verlag London, Ltd., London, 2008.

[8] F. Dumortier, J. Llibre, and J. C. Artés. Qualitative theory of planar differential systems. Universitext. Springer-Verlag, Berlin, 2006.

[9] A. F. Filippov. Differential equations with discontinuous righthand sides, volume 18 of Mathematics and its Applications (Soviet Series). Kluwer Academic Publishers Group, Dordrecht, 1988. 
[10] E. Freire, E. Ponce, F. Rodrigo, and F. Torres. Bifurcation sets of continuous piecewise linear systems with two zones. Internat. J. Bifur. Chaos Appl. Sci. Engrg., 28(11):20732097, 1998.

[11] E. Freire, E. Ponce, and F. Torres. Canonical discontinuous planar piecewise linear systems. SIAM J. Appl. Dyn. Syst., 11(1):181-211, 2012.

[12] M. Han and W. Zhang. On Hopf bifurcation in non-smooth planar systems. J. Differential Equations, 248(9):2399-2416, 2010.

[13] Y. Ilyashenko. Centennial history of Hilbert's 16th problem. Bull. Amer. Math. Soc. (N.S.), 39(3):301-354, 2002.

[14] J. Llibre and E. Ponce. Three nested limit cycles in discontinuous piecewise linear differential systems with two zones. Dyn. Contin. Discrete Impuls. Syst. Ser. B Appl. Algorithms, 19(3):325-335, 2012.

[15] J. Llibre, E. Ponce, and F. Torres. On the existence and uniqueness of limit cycles in Liénard differential equations allowing discontinuities. Nonlinearity, 21(9):2121-2142, 2008.

[16] R. Lum and L. O. Chua. Global properties of continuous piecewise-linear vector fields. Part I: Simplest case in $R^{2}, 1990$. Memorandum UCB/ERL M90/22, University of California at Berkeley.

[17] Z. F. Zhang, T. R. Ding, W. Z. Huang, and Z. X. Dong. Qualitative theory of differential equations, volume 101 of Translations of Mathematical Monographs. American Mathematical Society, Providence, RI, 1992. 